\title{
Crystal-melt interface mobility in bcc Fe: Linking molecular dynamics to phase-field and phase-field crystal modeling
}

\author{
M. Guerdane ${ }^{1}$ and M. Berghoff ${ }^{2}$ \\ ${ }^{1}$ Institute of Applied Materials, Computational Materials Science, Karlsruhe Institute of Technology (KIT), Germany \\ ${ }^{2}$ Steinbuch Centre for Computing, Karlsruhe Institute of Technology (KIT), Germany
}

(Received 19 December 2017; revised manuscript received 23 March 2018; published 12 April 2018)

\begin{abstract}
By combining molecular dynamics (MD) simulations with phase-field (PF) and phase-field crystal (PFC) modeling we study collision-controlled growth kinetics from the melt for pure Fe. The MD/PF comparison shows, on the one hand, that the PF model can be properly designed to reproduce quantitatively different aspects of the growth kinetics and anisotropy of planar and curved solid-liquid interfaces. On the other hand, this comparison demonstrates the ability of classical MD simulations to predict morphology and dynamics of moving curved interfaces up to a length scale of about $0.15 \mu \mathrm{m}$. After mapping the MD model to the PF one, the latter permits to analyze the separate contribution of different anisotropies to the interface morphology. The MD/PFC agreement regarding the growth anisotropy and morphology extends the trend already observed for the here used PFC model in describing structural and elastic properties of bcc Fe.
\end{abstract}

DOI: 10.1103/PhysRevB.97.144105

\section{INTRODUCTION}

The use of multiscale modeling is motivated by the fact that many phenomena in materials science and engineering involve interactions between microscopic and macroscopic length and time scales $[1,2]$. One of the central challenges of multiscale modeling is how to achieve the coupling between atomistic and macroscopic approaches to ensure that the descriptions at all levels remain quantitatively consistent with one another. This task is carried out in this work for the hierarchical coupling approach that combines molecular dynamics (MD) with phase-field (PF) simulations. We test the consistency by achieving detailed comparisons of quantitative predictions of the considered approaches for a typical multiscale problem: the solidification and melting kinetics.

Our way of proceeding consists of two steps: (1) Analyze how the phenomenological PF model can be properly designed in order to reproduce quantitatively the solidification kinetics observed by MD. The latter atomistic approach, considered as the reference, provides all the physical parameters needed to construct the former continuous one. (2) Once a consistent $\mathrm{MD} / \mathrm{PF}$ hybrid model is constructed, we use it for a better understanding of the growth kinetics, going further than would one single approach. The consistency analysis is aimed at continuously improving the design of the PF model and, consequently, enhancing the predictive accuracy of the MD/PF coupling.

We already applied this approach for an understanding of the thermodynamics and kinetics during growth of bcc $\mathrm{Zr}$ from an undercooled NiZr melt under chemical nonequilibrium [3]. We showed that the PF approach describes the same aspects of physics as MD, when the key parameters (like free energy, diffusivity, and interface properties) are transferred from MD to PF. Moreover, the effective thermodynamic enhancement of the diffusivity through the strong negative enthalpy of mixing in the NiZr solution was quantified. In another study [4], we applied the same coupling approach to capture, quantitatively, the correlation between the short-range order in the melt, the in-plane ordering (coupled to a diffusion drop) at the crystal-liquid interface, and the stability of the liquid against crystallization. In both studies [3,4], we dealt with the case of solidification under chemical nonequilibrium where the growth kinetics is dominated by the long-range-diffusion. In this study, we consider, however, the case of collisionlimited solidification which is controlled by the attachment rate of liquid atoms to the crystal surface. Consequently, solid-liquid (SL) anisotropies are expected to play a more significant role than for the diffusion-controlled solidification. The collision-limited growth is typical for pure and stoichiometric phases. We illustrate it here for pure Fe and investigate, thereby, the effect of interface anisotropies with regard to the interfacial energy $\gamma$ (capillarity), the kinetic coefficient $\mu$ (interface mobility), and, possibly, the interface thickness $\varepsilon$. It is worth noticing that understanding the interplay of $\gamma$ and $\mu$ anisotropies is a prerequisite to predict the dynamics of interface morphologies like dendrites and eutectics [5,6]. The latter pattern control the properties and, hence, the functionality of the final solidification product. In general, and due to the difficulty to get $\gamma$ - and $\mu$-anisotropy measurements, one still relies on atomistic simulation methods to provide these data or any insight into the physics of SL interfaces (and grain boundaries). In MD simulations, the SL interface anisotropies arise naturally as a product of the interatomic interaction. They are, however, intrinsically coupled and can hardly be separated from one another. In the PF approach, these anisotropies are included per construction and connected in a phenomenological way. The MD/PF coupling allows, on the one hand, to assess the phenomenological construction of the PF model. On the other hand, it permits to separate the role of each anisotropy independently. Berghoff et al. [7] already investigated the solidification of pure Ni linking MD calculations to PF ones. In an earlier pioneer work, Bragard 
et al. [6] presented a PF study of rapid solidification in undercooled liquid nickel [8] based on $\gamma$ and $\mu$ anisotropies calculated by MD. Growth rates and morphological evolution of dendrites were found in well agreement with experimental data as well as the predictions of the solvability theory.

In our present PF simulations, we use the $\gamma$ anisotropy of Fe that was predicted before by MD simulations [8]. The kinetic coefficient $\mu$ and its anisotropy will be here revisited to account for thermostat issues that have been neglected before [8] causing a significant underestimate of $\mu$ (by almost a factor of 2).

A further comparison will be achieved between the MD growth results and those from an atomistic phase-field crystal (PFC) model. The PFC approach emerged recently as a new promising research direction in the development of multiscale models [9]. The MD/PFC comparison can be considered here only for relative quantities, like growth-anisotropy parameters, whereas growth velocities cannot be directly compared. This is mainly because MD simulations treat collision-controlled solidification driven by undercooling (solid and liquid keep homogeneous atomic densities), whereas the PFC modeling describes a diffusion-controlled growth driven by density gradients. The growth velocity is constant in the first case while it decreases with time in the second one.

\section{MODELING METHODS}

\section{A. Molecular dynamics simulations}

We perform large-scale MD simulations for an isothermalisobaric $(N, T, p)$ ensemble of $N$ (up to $\sim 3 \times 10^{6}$ ) atoms, zero pressure $p$, and periodic boundary conditions in the three directions of space. We use the Fe interatomic potential developed by Mendelev et al. [10] (used also in Ref. [8]).

\section{Planar solid-liquid interface}

In order to create a planar SL interface, we start with a crystalline sample, the half of which (along one axis) is molten by heating it at a high temperature, while the other half is kept at its initial configuration. Then, the whole layer system is annealed at a temperature of interest for several picoseconds and, subsequently, production runs are carried out. The melting temperature $T_{m}$ is defined as the temperature at which the interface velocity $V_{I}$ vanishes. The crystal grows below $T_{m}$ and melts above. A fit of the relation $V_{I}=\mu\left(T_{m}-T\right)$ yields the kinetic coefficient $\mu$. In all the directional solidification simulations, only the box length normal to the interface is allowed to change. The two-phase samples have a rectangular cross section with sides corresponding to about 10-20 times $a_{0}$, the lattice constant of bcc Fe. Based on results of many MD works performed to date $[8,11]$, we can say that these box lengths are beyond the range where finite-size effects can be expected. The samples have a thickness of 50-150 times $a_{0}$ along the direction normal to the interface. For a better statistics of the calculated interface velocities, each growth or melting simulation is repeated 2-3 times starting from different configurations. The average is then achieved over the 4-6 resulting growth or melting fronts.

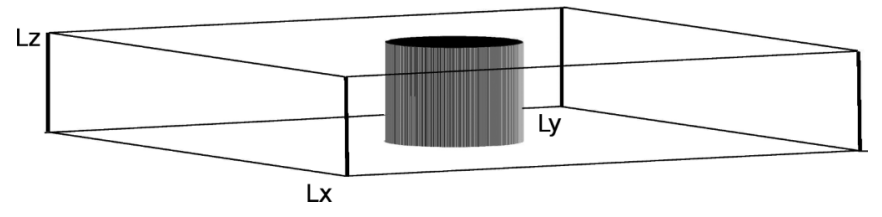

FIG. 1. Cylindrical crystalline seed that can grow freely in all directions perpendicular to the cylinder axis. We choose the cylinder axis parallel to the Cartesian direction $z$. The cylinder length is equal to the simulation-box length in that direction $L_{z}$.

\section{Cylindrical solid-liquid interface}

The analysis of interfacial anisotropies requires the simulation of curved interfaces, which call for MD systems much larger than those used for planar interfaces. The increasing computational capabilities make nowadays accessible such large-scale MD simulations $\left(10^{6}-10^{7}\right.$ atoms), at least for high growth velocities typical for collision-limited kinetics. We consider pseudo-two-dimensional solidification by setting up cylindrical crystalline seeds which can grow freely in all directions contained in the plane $x y$ (Fig. 1). During the growth simulations, the box lengths $L_{x}$ and $L_{y}$ are allowed to change while $L_{z}$ is kept at its equilibrium value. Along the $z$ Cartesian direction, the length of the cylindrical seed is set up equal to the simulation-box dimension $L_{z}$. Because of the periodic boundary conditions, the cylindrical SL interface remains parallel to the $z$ axis. $L_{z}$ is chosen as small as possible (6-12 times $a_{0}$ ), which allows the use of much larger box dimensions in $x$ and $y$ directions. Anisotropy effects are analyzed up to a length scale of about $0.15 \mu \mathrm{m}(1500 \AA)$. Two crystal orientations are simulated in our study: cylinder axis $(z)$ along [100] and [110] crystal directions. The growth rate in a given SL front direction is determined by averaging over 2-3 simulations, added to the average over all equivalent crystal directions owing to symmetry considerations.

\section{Temperature control: Layered and rectangular-cell thermostats}

As discussed in Ref. [12], a special care has to be taken when determining SL interface velocities by means of MD methods. The application of a global thermostat to the simulation sample leads to the formation of temperature gradients at the interface during growth or melting. In MD methods, the electronic contribution to the thermal conductivity is absent, making the dissipation of the latent heat abnormally slow. We remedy this problem by using the layered thermostat approach presented in [12]. In the case of a planar SL interface, the simulation cell is divided into layers parallel to the interface. Each layer has its own thermostat maintained at the same undercooling by a simple velocity rescaling after every MD step. It has been shown in [12] that the layer thickness $\delta$ below which the growth-simulation results are not affected by the temperature control method is in the range of $20 \AA$. We use a layer thickness $\delta=15 \AA$ for the directional one-dimensional free solidification.

Concerning the pseudo-two-dimensional solidification, where the interfaces are no longer planar, the simulation box is divided into a lattice of small rectangular cells of volume $l_{x} l_{y} l_{z}$ ( $l_{z}$ can be chosen here equal to $L_{z}$ ). Each cell has its thermostat 
fixed at the same temperature. Similar to the layered thermostat above, we choose thermostat cell lengths $l_{x}$ and $l_{y}$ in the range of $15 \AA$. This allows to maintain a nearly homogeneous temperature field in the growth plane $x y$. It is worth mentioning that the cell volume $V_{\text {cell }}=l_{x} l_{y} l_{z}$ has to be chosen large enough to ensure that the temperature is properly described within each rectangular cell and $T_{m}$ remains constant. Following statistical mechanics, the kinetic temperature of a system of $Z$ particles has a standard deviation $\sigma=\left(\sqrt{\left\langle T^{2}\right\rangle-\langle T\rangle^{2}}\right)=$ $T_{\text {bath }} \sqrt{2 /(3 Z)}$, where $T_{\text {bath }}$ is the target temperature [13]. Thus, the number of atoms in each cell must be large enough to keep the temperature fluctuations at a reasonable level.

\section{B. Phase-field modeling}

For the PF simulations, we use the phase model described in [14]. We start from an entropy functional defined on a spatial domain $\Omega$ :

$$
\mathcal{S}=\int_{\Omega}\left(s(\phi, T)-a(\nabla \phi)-\frac{1}{\varepsilon} w(\phi)\right) d \Omega,
$$

which contains separate bulk contributions $s(\phi, T)$ as a function of temperature and phase field $\phi$. The latter is an order parameter that distinguishes the solid from the liquid phase in our case. The gradient term $a(\nabla \phi)=\frac{1}{T \gamma_{0}} \varepsilon|\gamma(\hat{\mathbf{n}}) \nabla \phi|^{2}$ controls traditionally the interfacial energy $\gamma(\hat{\mathbf{n}})$, made anisotropic by writing $\gamma(\hat{\mathbf{n}})=\gamma_{0} A(\hat{\mathbf{n}})$, where $\gamma_{0}$ is the mean value and $A(\hat{\mathbf{n}})$ a dimensionless anisotropy function depending on the interface normal vector $\hat{\mathbf{n}}=\frac{\nabla \phi}{|\nabla \phi|}$. A SL interface thickness is given by $2 \varepsilon$. For $w(\phi)$, we choose the double-well potential $w(\phi)=$ $9 \gamma_{0} \phi^{2}(1-\phi)^{2}$ with two minima representing the liquid and solid phases.

The evolution equation for the phase field $\phi$ follows from a local maximization of the entropy functional of Eq. (1) using a variational approach:

$$
\begin{aligned}
\varepsilon \tau \frac{\partial \phi}{\partial t}= & \nabla \cdot\left(\frac{\partial}{\partial \nabla \phi}-\frac{\partial}{\partial \phi}\right) a(\nabla \phi) \\
& -\frac{1}{\varepsilon} \frac{\partial w(\phi)}{\partial \phi}-\frac{1}{2 T}\left(f^{s}-f^{l}\right) \frac{\partial h(\phi)}{\partial \phi} .
\end{aligned}
$$

$f^{s}(T)$ and $f^{l}(T)$ denote the free-energy density of solid and liquid, respectively. $h(\phi)$ is a suitable interpolation function between the two phases. We use the polynomial $h(\phi)=$ $\phi^{3}\left(6 \phi^{2}-15 \phi+10\right)$.

The coefficient $\tau$ follows from an asymptotic analysis of the sharp-interface limit: $\tau(\hat{\mathbf{n}})=\frac{L}{\mu(T, \hat{\mathbf{n}}) T_{m} T}$. We use a $T$-dependent kinetic coefficient $\mu(T, \hat{\mathbf{n}})=\frac{V_{I}(T, \hat{\mathbf{n}})}{\Delta T}$ to account for the nonlinearity between the growth velocity $V_{I}$ and the undercooling $\Delta T$ (see Sec. III A).

For pure elements, $\left[f_{s}(T)-f_{l}(T)\right]$ can well be approximated by [7]

$$
\begin{aligned}
f^{s}(T)-f^{l}(T)= & \frac{L\left(T-T_{m}\right)}{T_{m}}+\Delta C_{p}\left(T-T_{m}\right) \\
& -T \Delta C_{p} \ln \left(\frac{T}{T_{m}}\right),
\end{aligned}
$$

\begin{tabular}{|c|c|c|c|}
\hline $\begin{array}{l}T_{m} \\
(\mathrm{~K})\end{array}$ & $\begin{array}{c}L \\
(\mathrm{eV} / \text { atom })\end{array}$ & $\begin{array}{c}C_{p}^{s} \\
{[\mathrm{meV} /(\mathrm{K} \text { atom })]}\end{array}$ & $\begin{array}{c}C_{p}^{l} \\
{[\mathrm{meV} /(\mathrm{K} \text { atom }]}\end{array}$ \\
\hline 1772 & 0.162 (Ref. [8]) & 0.333 & 0.353 \\
\hline
\end{tabular}

where $\Delta C_{p}=C_{p}^{s}-C_{p}^{l}$, the difference between the specific heat capacities of solid and liquid, respectively. MD values
TABLE I. Thermodynamic parameters calculated from the MD Fe model.

of $C_{p}^{s, l}$ are calculated from the slope of the enthalpy curve: $C p=d H / d T$ (see Table I). $L$ is the latent heat.

Equation (3) is readily deduced by relating the free energy $f=H-T S$ at an arbitrary temperature $T$ to that at $T_{m}$ by means of a thermodynamic integration

$$
\begin{aligned}
f^{s, l}(T)= & H^{s, l}\left(T_{m}\right)+C_{p}^{s, l}\left(T-T_{m}\right) \\
& -T\left(S^{s, l}\left(T_{m}\right)+C_{p}^{s, l} \int_{T_{m}}^{T} \frac{d T^{\prime}}{T^{\prime}}\right)
\end{aligned}
$$

and considering the enthalpy and entropy jump at $T_{m}$ : $H^{l}\left(T_{m}\right)-H^{s}\left(T_{m}\right)=L ; S^{l}\left(T_{m}\right)-S^{s}\left(T_{m}\right)=L / T_{m}$. The first term of Eq. (3) expands the difference between liquid and solid free energies near $T_{m}$. The second and third terms represent a correction taking into account that the enthalpy curves $H^{s}(T)$ and $H^{l}(T)$ do not run parallel to one another.

Despite we deal with the case of isothermal growth by setting $T$ constant everywhere, curved interfaces are nonisothermal owing to the Gibbs-Thomson effect. This latter is naturally included in MD simulations. In order to account for this effect in the PF simulations, the undercooling at the interface is implicitly replaced by an effective one according to the equation $[5,6]$

$$
(\Delta T)_{\mathrm{eff}}=T_{m}-T-\frac{T_{m}}{L} \sum_{i=1,2}\left(\gamma(\hat{\mathbf{n}})+\frac{\partial^{2} \gamma(\hat{\mathbf{n}})}{\partial \theta_{i}^{2}}\right) \frac{1}{R_{i}},
$$

where the bracketed term denotes the interface stiffness. $R_{i}$ $(i=1,2)$ are the principal radii of curvature and $\theta_{i}$ two principal local angles fixing the interface.

\section{RESULTS}

\section{A. MD and phase-field simulations}

Concerning the free energy $\gamma(\hat{\mathbf{n}})$, we use the calculations of Sun et al. [8]. For systems with a cubic symmetry, the anisotropy of a quantity $q(\hat{\mathbf{n}})$ can be accurately described using two parameters $\delta_{1}$ and $\delta_{2}$ defined by an expansion in terms of cubic harmonics [15]:

$$
\frac{q(\hat{\mathbf{n}})}{q_{0}}=1+\delta_{1}\left(Q-\frac{3}{5}\right)+\delta_{2}\left(3 Q+66 S-\frac{17}{7}\right),
$$

with $Q=n_{1}^{4}+n_{2}^{4}+n_{3}^{4}$ and $S=n_{1}^{2} n_{2}^{2} n_{3}^{2}$. The $n_{i}$ 's are the components of the interface normal $\hat{\mathbf{n}}$. The same expansion function is assumed to describe the anisotropy of $\gamma(\hat{\mathbf{n}})$ and $\mu(\hat{\mathbf{n}})$. The expansion parameters used in the PF model are listed in Table II.

To characterize the crystal-to-liquid transition, we choose to use the fine-grained number-density profile (using about 10 bins per $\AA$ ). We justify this choice at the end of the present section. The results of this MD analysis are illustrated in 
TABLE II. Anisotropic quantities $\gamma(\hat{\mathbf{n}})$ (in $\mathrm{mJ} / \mathrm{m}^{2}$ ) and $\mu(\hat{\mathbf{n}})$ (in $\mathrm{m} / \mathrm{s} \mathrm{K}$ ) with their anisotropy parameters $\delta_{1}$ and $\delta_{2}$ according to Eq. (6). For $\mu$, we used $\mu(T, \hat{\mathbf{n}})=\frac{V_{I}(T, \hat{\mathbf{n}})}{\Delta T}$, where $V_{I}$ are the velocities in Fig. 3.

\begin{tabular}{lcccccr}
\hline \hline & $q_{100}$ & $q_{110}$ & $q_{111}$ & $q_{0}$ & $\delta_{1}$ & \multicolumn{1}{c}{$\delta_{2}$} \\
\hline$\gamma$ (Ref. [8]) & 177.0 & 173.5 & 173.4 & 174.5 & 0.033 & 0.0025 \\
$\mu\left(T_{m}\right)$ & 0.774 & 0.677 & 0.668 & 0.702 & 0.235 & 0.0136 \\
$\mu(\Delta T=45 \mathrm{~K})$ & 0.691 & 0.634 & 0.611 & 0.644 & 0.184 & -0.0025 \\
\hline \hline
\end{tabular}

Fig. 2 for the (110) SL interface. The decaying amplitude of the density wave is taken as a structural order parameter. We determine the interfacial width from a fitting of the density maxima by a hyperbolic tangent function:

$$
\eta(x)=\frac{\left(\rho_{l}+\eta_{s}\right)}{2}+\frac{\left(\rho_{l}-\eta_{s}\right)}{2} \tanh \left(\frac{3\left(x-x_{0}\right)}{2 \varepsilon}\right) .
$$

$\eta_{s}$ fits the average density maximum in the solid, while $x_{0}$ fits the mean position of the interface. The average liquid density $\rho_{l}$ is determined separately and used as a fixed parameter in the fit. The function (7) is physically founded as it is the equilibrium solution of Eq. (2) at $T_{m}$ for a planar SL interface using the double-well potential $w(\phi)$ [16]. The fitting procedure yields an interface width averaged over the three low-index directions $2 \bar{\varepsilon}=16.89 \AA$. This value is used in our PF simulations below.

Figure 3 exhibits interface velocities $V_{I}$ versus temperature for planar solidification and melting for the low-index orientations. The melting temperature $T_{m}$ follows from the intersection of the fitted $V_{I}$ curves with the $T$ axis. An averaging over the three interface orientations yields $T_{m}=1772.3 \pm 1.5 \mathrm{~K}$, which is the same as the $T_{m}$ value in Refs. $[8,10]$. For the determination of the kinetic coefficient $\mu$ in the small undercooling range, about 10 temperatures within the interval $[1750,1790] \mathrm{K}$ are used for a linear fit $V_{I}=\mu\left(T_{m}-T\right)$. We obtain $\mu_{100}=77.4 \pm 1.7 \mathrm{~cm} / \mathrm{s} \mathrm{K}, \mu_{110}=67.7 \pm 1.4 \mathrm{~cm} / \mathrm{s} \mathrm{K}$, and $\mu_{111}=66.8 \pm 1.7 \mathrm{~cm} / \mathrm{s} \mathrm{K}$. The data of this analysis can be found in the Supplemental Material [17]. For larger undercooling, we use a polynomial fit of the data (dashed lines in Fig. 3). The anisotropies $\mu_{100} / \mu_{110}$ and $\mu_{100} / \mu_{111}$ are 1.15

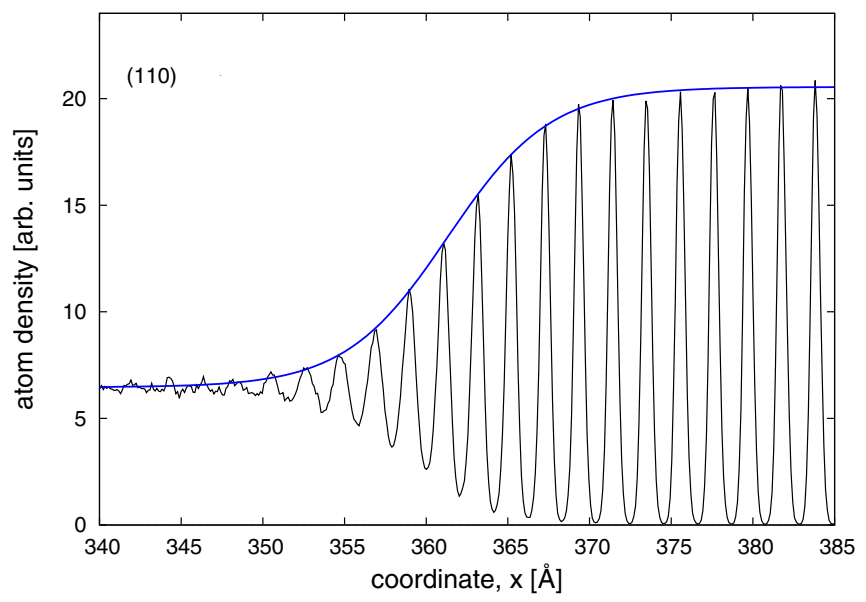

FIG. 2. Fine-grained density profile for (110) SL interface near $T_{m}$. The solid line is a least-squares fit using a hyperbolic tangent function of Eq. (7).

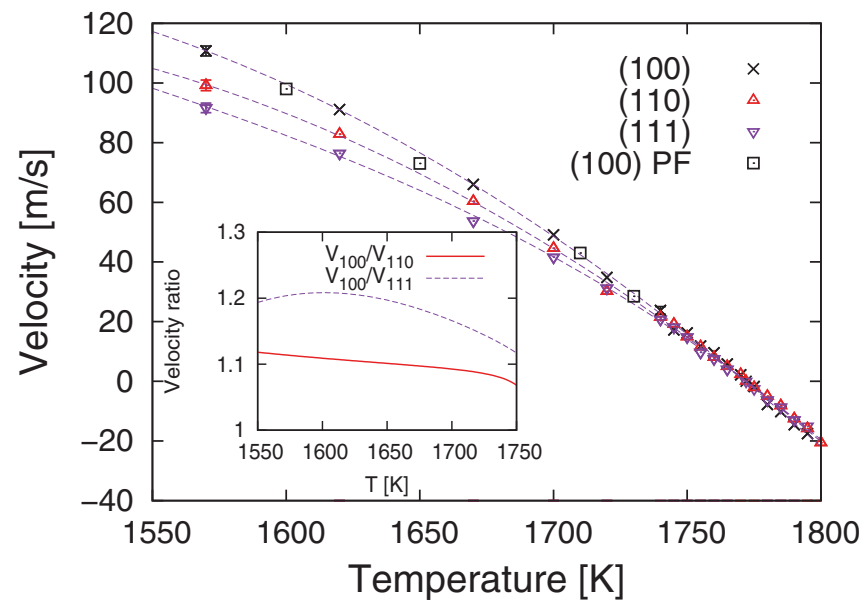

FIG. 3. MD velocities of planar SL interfaces as a function of $T$. Positive (versus negative) velocities correspond to growth (versus melting). The dashed lines are least-squares fits to the data. The standard errors are illustrated for the largest undercooling only. PF data for (100) are included. Inset: growth-rate anisotropy for large undercooling calculated by using the fitting curves. Regarding the calculation of the kinetic coefficient $\mu$ near $T_{m}$, a linear fit was used (see text).

and 1.16, respectively. These results are comparable to those obtained by Gao et al. [18] for the same Fe potential model using a global temperature thermostat but considering the local interface temperature to determine the actual undercooling. This agreement confirms the conclusion made by Monk et al. [12] about the equivalence between the layered thermostat technique, we use here, and that relying on the true interface temperature. We notice that earlier MD works, which applied a global thermostat, came to smaller $\mu$ values (by about a factor 2 ) and to overestimated anisotropies $[8,19]$.

As illustration, Fig. 3 includes a few interface velocities from PF calculations for the (100) orientation by solving Eq. (3). This very good concordance between MD and PF results was found for the three low-index orientations of the interface. This shows that the PF model can be properly parametrized to reproduce quantitatively the collision-limited solidification dynamics given by MD modeling. This confirms the agreement we already observed in the case of diffusioncontrolled growth in a binary alloy (NiZr) under chemical nonequilibrium [3]. In return, this agreement assesses the ability of MD modeling to treat correctly solidification kinetics driven by thermodynamic forces. Equation (2) confirms that the thermodynamic driving force of freezing can be represented by $f_{s}-f_{l}$, that is, the difference between the free energies of solid and liquid. In the case of diffusion-determined growth [3], the growth kinetics was found to depend on the difference between the chemical potentials (derivative of the free energies) of the individual species in the solution. The MD/PF agreement in Fig. 3 concerns, however, only directional velocities and says nothing about reproducing or not the anisotropy effects that are, namely, not active for planar-interface growth. The situation is different for the following cylindrical interfaces.

Figure 4 compares cross-section snapshots during the growth of initial cylindrical seeds from $\mathrm{MD}$ and $\mathrm{PF}$ 

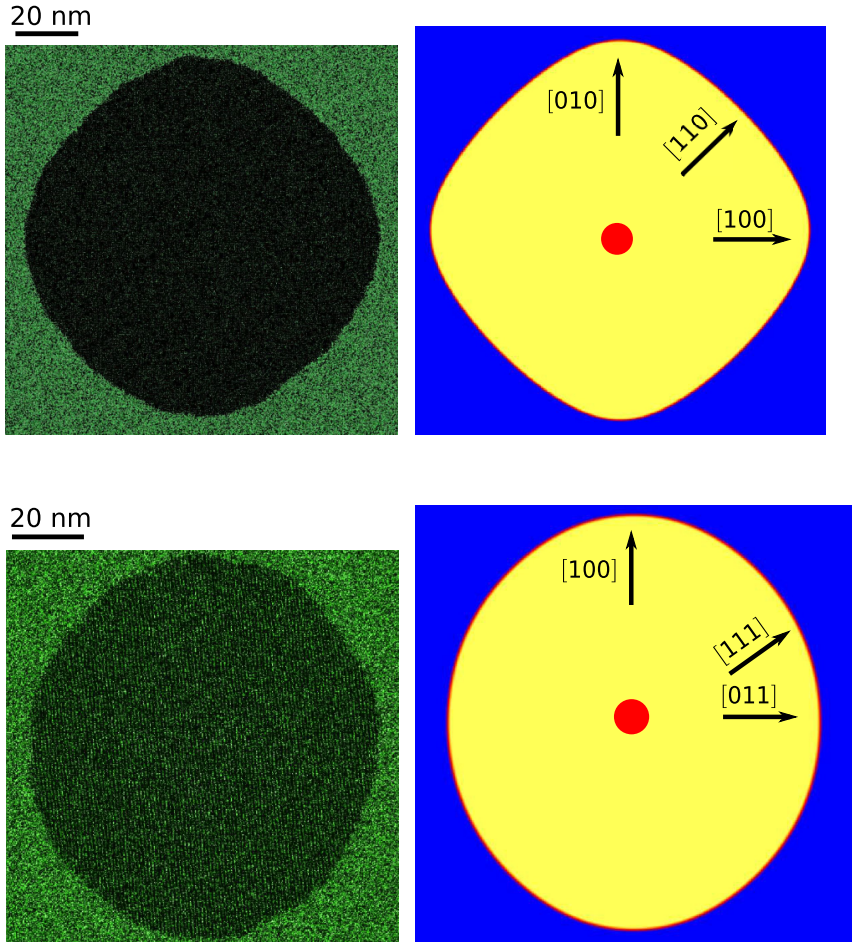

FIG. 4. Snapshots from MD and PF simulations at $T=1600 \mathrm{~K}$ which illustrate the growth of cylindrical crystalline samples. Crystal orientation: cylinder axis along [100] (top) and [110] (bottom). Simulation time: 585 and 560 ps, respectively. MD-sample size: 2613124 atoms in $(1374.4 \times 1374.4 \times 17.4) \AA^{3}$ and 2558500 atoms in $(1268.1 \times 1271.2 \times 20.7) \AA^{3}$, respectively. The initial seeds of diameters $D_{0}=90$ and $140 \AA$ are indicated by the red disks at the centers of the PF samples. The shown length scale concerns MD and PF samples.

simulations for [100] (top) and [110] (bottom) orientations of the cylinder axis. The samples exhibit clearly a fourfold and twofold symmetry, respectively, with a preferential growth in (100)-interface front relative to (110) one. These growth symmetries are reproduced with the same degree of perfectness for all MD simulations we achieved at different undercoolings. This fact is remarkable when we keep in mind that the curved interfaces are constructed atom by atom. The sharper curvature in $\langle 100\rangle$ direction presages the dendrites branches which would develop at larger space and timescales (and nonisothermal setup), conditions which are still beyond the capabilities of atomistic modeling. In the following, we analyze the effect of $\gamma(\hat{\mathbf{n}})$ and $\mu(\hat{\mathbf{n}})$ anisotropies on the growth rate and morphology. For this sake, we represent in Figs. 5 and 6 the position-time relationship $\Delta R(t)=\left[D(t)-D_{0}\right] / 2$ for the growth front along the (100)- and (110)-interface fronts for an undercooling $\Delta T=45 \mathrm{~K} . D(t)$ is the corresponding diameter of the seed at time $t, D_{0}$ is the diameter of the initial cylindrical seed. Common to MD and PF results is the increase of the growth velocity [slope of $\Delta R(t)$ ] with time. This reflects the Gibbs-Thomson effect which becomes weaker as the interface curvature decreases with growth. After $t=1 \mathrm{~ns}$, the (100)-interface front propagates with a velocity of about $300 \AA / \mathrm{ns}(30 \mathrm{~m} / \mathrm{s})$ which is comparable with the velocity

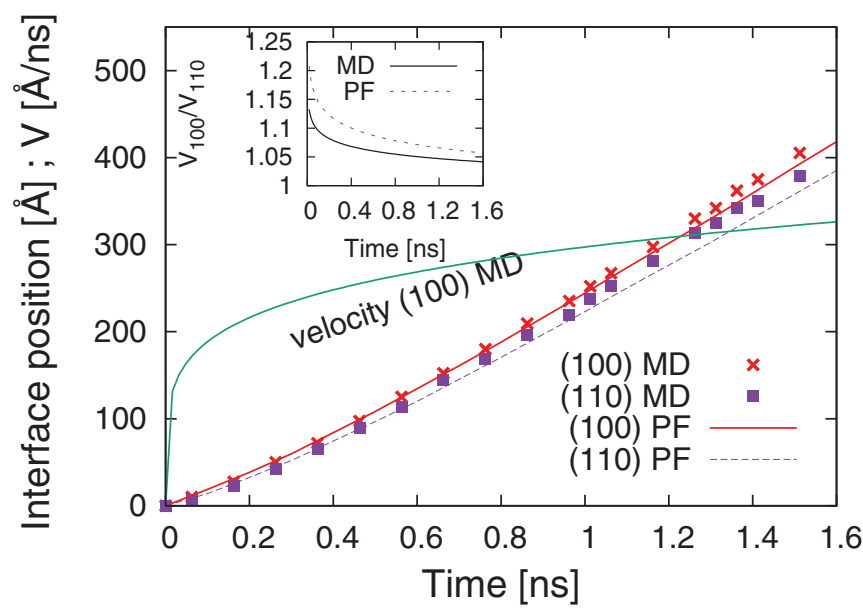

FIG. 5. Front position $\Delta R(t)$ along the [100] and [110] directions for a cylinder-axis orientation along [100] (top of Fig. 4). MD results are compared with PF ones at an undercooling of $\Delta T=45 \mathrm{~K}$. Inset: growth-rate anisotropy. The velocity functions are obtained by the derivative of the polynomial fits of $\Delta R(t)$.

$31.1 \mathrm{~m} / \mathrm{s}$ of a planar (100) interface at the same undercooling in Fig. 3. The anisotropy $V_{100} / V_{110} \sim 1.05$ (inset of Fig. 5) is smaller than the value $\sim 1.1$ for planar interfaces in Fig. 3 . This anticipates the conclusion we will draw below, namely, that the growth morphology can not be rationalized only by the anisotropy of the kinetic coefficient $\mu(\hat{\mathbf{n}})$.

The Gibbs-Thomson effect on the growth velocity becomes weaker at much larger undercoolings as depicted in Fig. 7 for $\Delta T=175 \mathrm{~K}$. The curvature undercooling becomes only a neglectible fraction of the interface undercooling which can be, then, assimilated to the kinetic one $\left[(\Delta T)_{\text {eff }} \sim \frac{V_{I}(T, \hat{\mathbf{n}})}{\mu(T, \hat{\mathbf{n}})}\right]$. As a consequence, the approximately constant-velocity regime is faster reached at larger undercoolings. These observations confirm the known fact that the interface dynamics is kinetics (versus capillary) dominated at high (versus low) undercoolings [6].

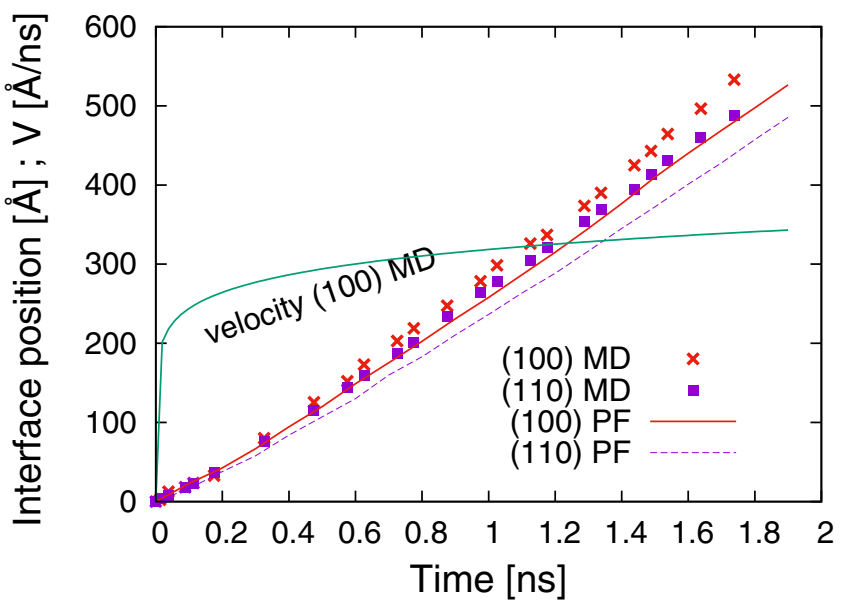

FIG. 6. As in Fig. 5 for a cylinder-axis orientation along [110] (bottom of Fig. 4). 


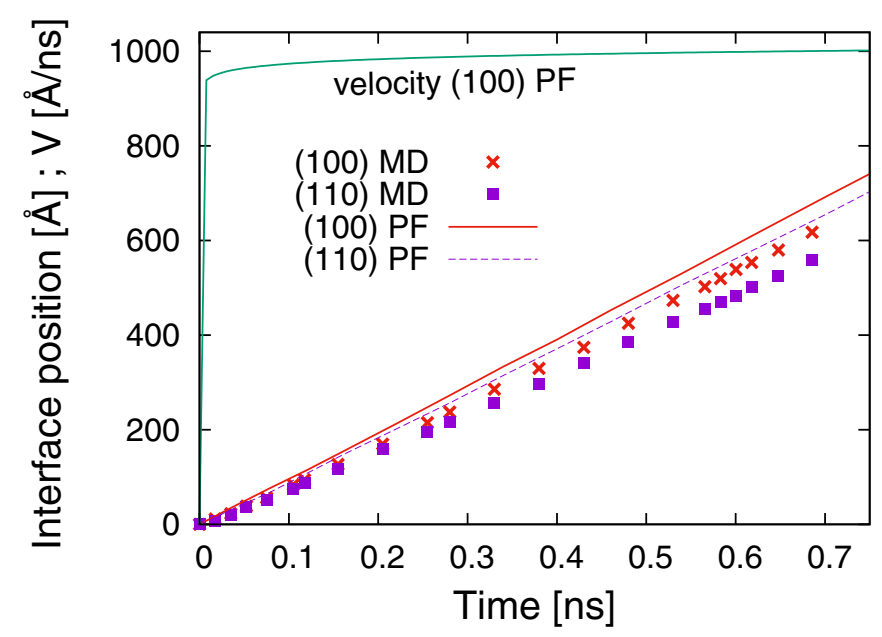

FIG. 7. As in Fig. 5 for a cylinder-axis orientation [100] at an undercooling $\Delta T=172 \mathrm{~K}$.

Relative to the MD approach, PF modeling has the advantage to allow a quantitative analysis of how a given physical parameter separately affects the growth process. This possibility is not given in MD modeling since all contributions are intrinsically and inseparably included in the interatomic interaction. However, after MD modeling has provided the PF model with the necessary physical parameters, it benefits from this model to identify the role of each parameter independently. We used this particularity to study the influence of $\gamma(\hat{\mathbf{n}})$ and $\mu(\hat{\mathbf{n}})$ anisotropies on the observed growth morphology. Figure 8 exhibits results of three growth simulations, where either both anisotropies are activated or one of them is "on" and the other is "off." The latter simulation condition is obviously hypothetical and is likely nonphysical because $\gamma(\hat{\mathbf{n}})$ and $\mu(\hat{\mathbf{n}})$
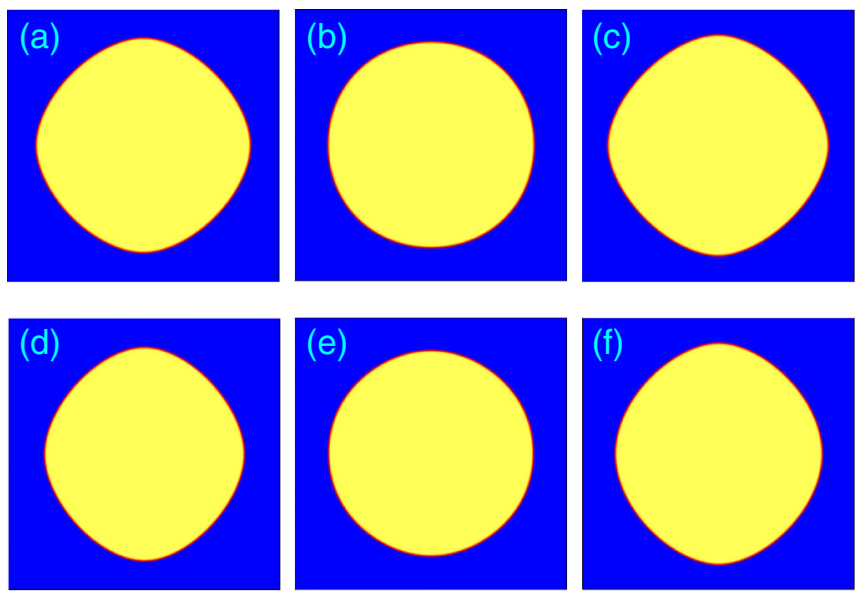

FIG. 8. Results of different PF growth simulations at an undercooling $\Delta T=75 \mathrm{~K}$ after $0.5 \mathrm{~ns}$, starting from cylindrical seeds. Top [(a)-(c)]: cylinder-axis orientation along [100]. Bottom [(d)(f)]: cylinder-axis orientation along [110]. In (a) and (d), $\gamma$ and $\mu$ anisotropies are activated. In (b) and (e), only the $\mu$ anisotropy is activated, while $\gamma(\hat{\mathbf{n}})=\gamma_{0}$. In (c) and (f), only $\gamma$ anisotropy is activated, while $\mu(\hat{\mathbf{n}})=\mu_{0}$. All simulations are carried out starting from cylindrical seeds of diameter $D_{0}=40 \AA$. The simulation box has a side of about $600 \AA$. anisotropies are thought to be dependent. This dependency has been poorly investigated $[5,6]$. Figure 8 demonstrates clearly that the fourfold and twofold morphology is controlled by the capillarity $\gamma(\hat{\mathbf{n}})$ anisotropy. When only $\mu(\hat{\mathbf{n}})$ anisotropy is activated $\left[\mu(\hat{\mathbf{n}}) / \mu_{0}=1\right]$, the sample keeps almost its initial cylindrical shape. We conclude that the growing seed is shaped rather by the anisotropic tension $\gamma(\hat{\mathbf{n}})$ that acts on the SL interface. $\mu(\hat{\mathbf{n}})$ anisotropy likely favors the existing morphology but does not generate it. This conclusion is not obvious to draw when using MD tools alone, as illustrated by the study [19] of growth simulations based on another Fe potential. The authors assigned the observed fourfold morphology to the $\mu(\hat{\mathbf{n}})$ anisotropy.

Our choice of the fine-grained density profile to characterize the SL interface was motivated by the idea to identify a possible interface-width anisotropy. The nature and extension of a SL interface remains a controversial issue in condensed-matter physics. Various order parameters expected to distinguish crystalline from liquid environment have been proposed in the literature (see [20] and references therein). Linking an atomistic method (MD) to a theoretical model (PF), where the interface thickness enters explicitly as a parameter, offers a physically reasoned way to say whether an order parameter is a good candidate for an objective, orientation-dependent, delimitation of the interface. We postpone this analysis to a future work since we realized that treating this issue would go beyond the scope of this paper. In order to account for a possible width anisotropy, as suggested by the fine-grained density representation, it is not enough to let $\varepsilon$ depend on the interface orientation in the evolution equation (3). We have to start at the level of the entropy functional of Eq. (2). The dependence of $\varepsilon(\hat{\mathbf{n}})$ of $\nabla \phi\left(\right.$ since $\hat{\mathbf{n}}=\frac{\nabla \phi}{|\nabla \phi|}$ ) has to be taken into account when applying the variational principle to derive the evolution equations and when achieving the asymptotic analysis of the sharp-interface limit.

\section{B. Phase-field crystal}

Instead of using spatially uniform or slow-varying phase fields of the traditional PF approach, PFC uses a local time average of the atomic density $\rho(\mathbf{r})$. The free-energy functional of a solid phase is minimized when the density field is periodic. Any disturbance or topological defect of the lattice leads to an increase of the free energy, capturing in this way information which is inherent only to atomistic approaches. This enables the PFC description to naturally incorporate anisotropy, crystal grain orientations, dislocations, and other lattice defects. The advantage of the PFC approach over the MD one is that, per construction, it integrates atomic vibrations on timescales many orders of magnitude larger than the time associated with the Debye frequency. Therefore, it is possible for the PFC methodology to work on diffusive timescales beyond a $\mu$ s. There are different formulations of the PFC model in the literature [9]. We use the model of Jaatinen et al. [21] based on an eighth-order fitting (EOF) of the two-body correlation function of liquid. As reference, the authors used the system described by the same MD model [10] in Sec. II A, which allows us a direct comparison of our MD and PFC calculations. The authors showed that this PFC model reproduces reasonable values of the crystal-melt interface energy $\gamma$, the 
TABLE III. Parameters of the PFC model after Jaatinen et al. [21].

\begin{tabular}{cccccccc}
\hline \hline$\rho_{0}$ & $k_{m}$ & $a$ & $b$ & $C_{0}$ & $\Gamma$ & $E_{B}$ & $u_{s}$ \\
\hline $0.0801 \AA^{-3}$ & $2.985 \AA^{-1}$ & 0.6917 & 0.0854 & -49 & 11.583 & 38.085 & 0.72 \\
\hline
\end{tabular}

SL coexistence gap, the bulk modulus, and the grain boundary energy of bcc Fe.

Defining the dimensionless density deviation $n(\mathbf{r})=$ $\frac{\left[\rho(\mathbf{r})-\rho_{0}\right]}{\rho_{0}}$, where $\rho_{0}$ is a uniform reference density, the freeenergy functional can be expressed as (we use the notation of [21])

$$
\frac{\Delta \mathcal{F}[n(\mathbf{r})]}{k_{B} T \rho_{0}}=\int\left\{\frac{1}{2} n(\mathbf{r}) \hat{\mathcal{C}} n(\mathbf{r})-\frac{a}{6} n(\mathbf{r})^{3}+\frac{b}{12} n(\mathbf{r})^{4}\right\} d \mathbf{r}
$$

with the operator $\hat{\mathcal{C}}=1-C\left(k_{m}\right)-\Gamma\left[\frac{k_{m}^{2}+\nabla^{2}}{k_{m}^{2}}\right]^{2}-E_{B}\left[\frac{k_{m}^{2}+\nabla^{2}}{k_{m}^{2}}\right]^{4}$.

We have $C(k)=1-1 / S(k) ; k_{m}$ is the position of the first peak of the static structure factor of liquid $S(k)$. The model parameters $a, b, \Gamma$, and $E_{B}$ are defined by $a=3\left[2 S\left(k_{m}\right) u_{s}\right]^{-1}, b=4\left[30 S\left(k_{m}\right) u_{s}^{2}\right]^{-1}, \Gamma=-\frac{1}{8} k_{m}^{2} C^{\prime \prime}\left(k_{m}\right)$, $E_{B}=C\left(k_{m}\right)-C_{0}-\Gamma . S(k)$ and $u_{s}$ are obtained from MD simulations of the same Fe model we use in this work [22]. The different parameters are listed in Table III. More details can be found in Ref. [21]. This model delivers the equilibrium coexistence densities $n_{l}=-0.025$ and $n_{s}=0.009$ for liquid and solid, respectively.

One-dimensional crystallization simulations are carried out using a planar SL layer system similar to that in Sec. II A 1. Pseudo-two-dimensional solidification is achieved using initial cylindrical crystalline seeds, similar to the setup described in Section II A 2. The initial liquid has a constant density, whereas the crystal part has a periodic structure that we realize here through the use of a one-mode approximation

$$
\begin{aligned}
n(\mathbf{r})= & 4 u_{s}(\cos (q x) \cos (q y)+\cos (q y) \cos (q z) \\
& +\cos (q z) \cos (q x)),
\end{aligned}
$$

where $u_{s}$ and $q$ are the amplitude and the wave number, respectively, of the density fluctuations around $n=0$. We start with an initial supersaturated liquid by setting its density $n$ higher than that deduced from the coexistence condition: $n>n_{l}=-0.025$. We recall that growth by PFC approaches is driven by a density gradient that builds in the liquid and becomes flatter with time. This situation differs from our simulations of $\mathrm{Fe}$ growth by MD where the density remains homogeneous in liquid and solid. This aspect has to be kept in mind when comparing PFC with MD results. Concerning the simulation methodology, growth driven by a density gradient requires to choose the simulation-box size along the growth direction large enough to avoid (otherwise minimize) the finite-size effects.

The PFC dynamics is assumed to be conservative and driven to minimize the functional of Eq. (8):

$$
\begin{aligned}
\frac{\partial n}{\partial t} & =M \nabla^{2} \frac{\delta(\Delta \mathcal{F})}{\delta n} \\
& =M \nabla^{2}\left(n(\mathbf{r}) \hat{\mathcal{C}} n(\mathbf{r})-\frac{a}{2} n(\mathbf{r})^{2}+\frac{b}{3} n(\mathbf{r})^{3}\right) .
\end{aligned}
$$

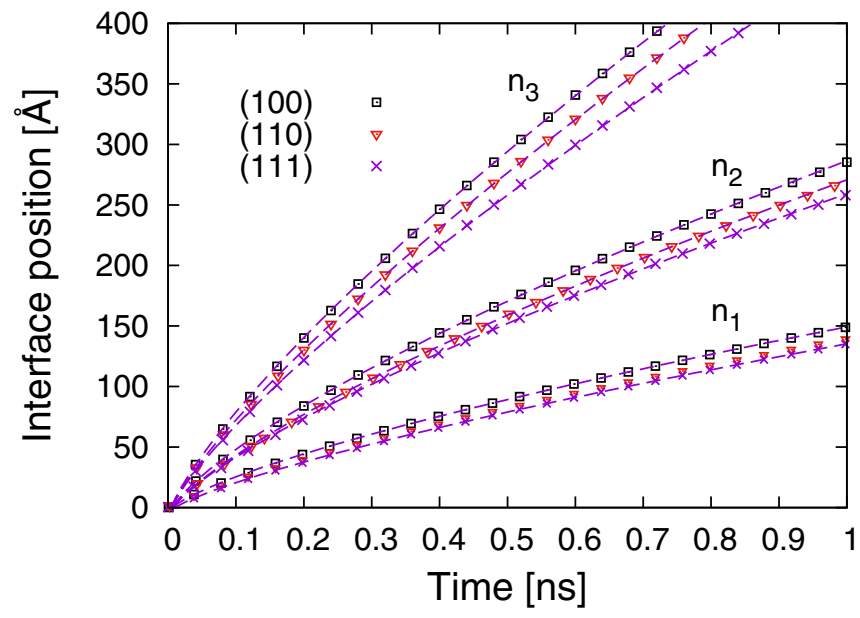

FIG. 9. Interface position versus time by PFC for the reduced densities $n_{1}=-0.00123, n_{2}=0.0048$, and $n_{3}=0.0131$. The data are fitted according to Eq. (11) (dashed lines).

The mobility parameter $M$ sets the timescale and depends, in general, on local density. For simplicity, we assume $M$ to be constant and set it to the value of the diffusivity in liquid Fe near the model melting temperature $T_{m}: M=10^{-9} \mathrm{~m}^{2} / \mathrm{s}$. Equation (10) is solved on a periodic regular grid using the semi-implicit spectral method suggested by Tegze et al. [23]. The time and spatial steps are $\Delta t=0.1 \mathrm{ps}$ and $\Delta x=\Delta y=\Delta z=a / 10 \simeq$ $0.29 \AA$, respectively.

Figure 9 depicts the position-time relationship of the (100), (110), and (111) SL interfaces during one-dimensional crystallization. The data are fitted to the function

$$
z(t)=C\left(t-t_{0}\right)^{m}+z_{0} .
$$

The exponent $m$ is found to be in the range of $0.71-0.74$ for all orientations and densities of Fig. 10. The latter exhibits the density-dependent growth velocity after $t^{\prime}=1 \mathrm{~ns}$. The quotients $\varepsilon_{1}=V_{100} / V_{110}$ and $\varepsilon_{2}=V_{100} / V_{111}$ (inset) give an idea about the growth anisotropy. We obtain similar $\varepsilon_{1}$ and $\varepsilon_{2}$

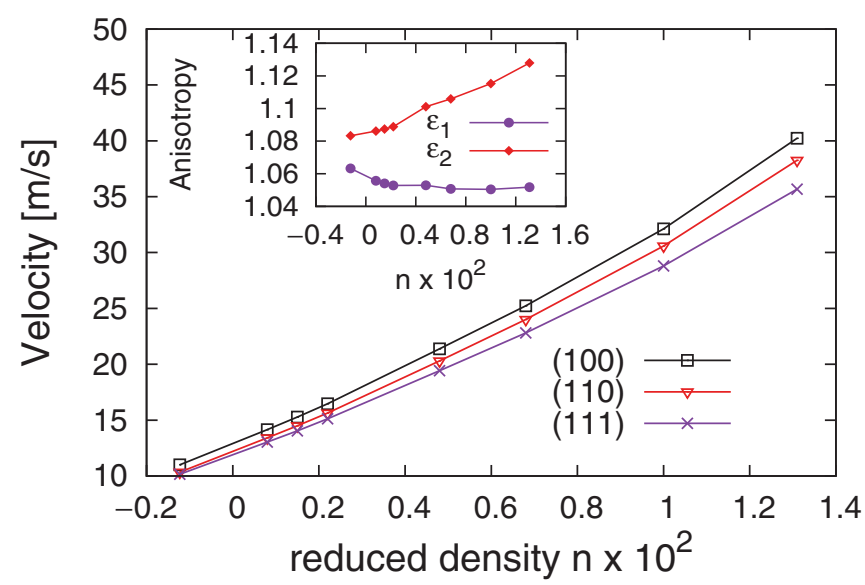

FIG. 10. Growth velocity versus density after $t^{\prime}=1 \mathrm{~ns}$, obtained by the derivative of the fitting functions in Fig. 9. Inset: anisotropy coefficients $\varepsilon_{1}$ and $\varepsilon_{2}$ (see text) versus density $n$. 


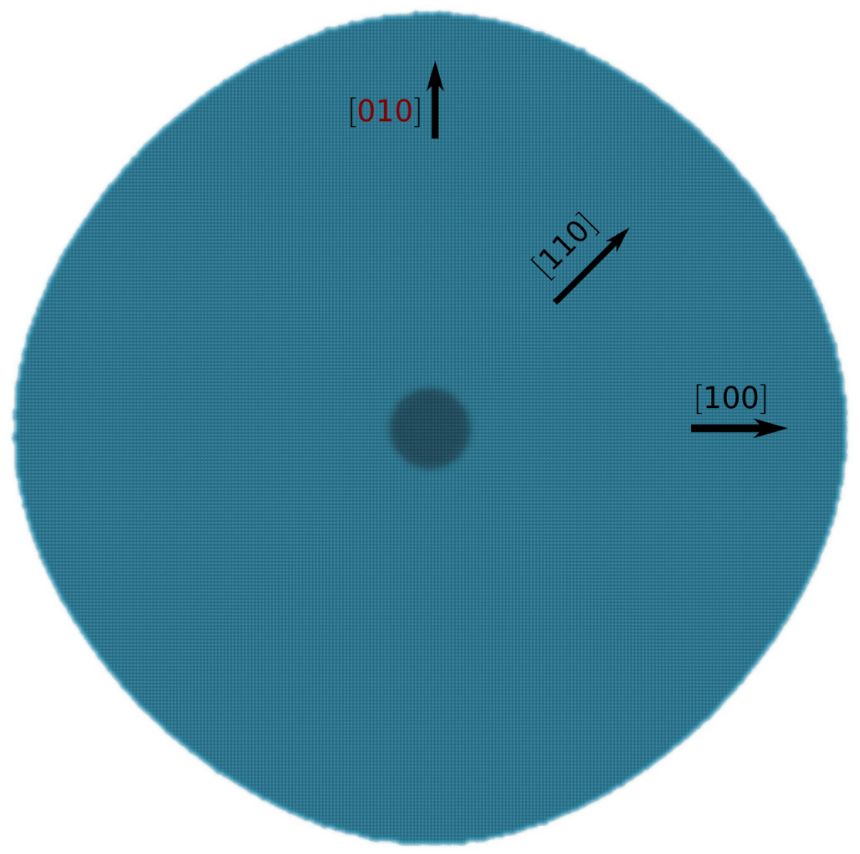

FIG. 11. PFC simulation snapshot during growth of a cylindrical crystalline sample. The initial seed has a diameter of 24 bcc cells (about $70 \AA$ ) and is indicated by the red disk at the center. Liquid density: $n=n_{3}=0.0131$. Crystal orientation: cylinder axis parallel to [100] (compare with top of Fig. 4). The sample has a thickness of three bcc elementary cells perpendicular to the figure plane. The atomic structure can be resolved by zooming in on the figure.

curves for other $t^{\prime}$ values or when defining the anisotropy as the quotient of the $C$ coefficients in Eq. (11) (this definition was used in the PFC studies [24,25]).

The deviation of the growth kinetics from a square-root behavior (i.e., $z \propto \sqrt{t-t_{0}}$ ) is somewhat unexpected owing to the fact that PFC models deal with a diffusion-controlled growth (driven by density differences). The PFC model in Ref. [25], which is based on a different parametrization of the free-energy functional of Eq. (8), predicts indeed an exponent $m=\frac{1}{2}$. We notice that a deviation of a diffusion-controlled growth from a square-root law is, though, not that strange. In our earlier work [4] regarding the growth kinetics of bcc $\mathrm{Zr}$ from a Ni-supersaturated $\mathrm{NiZr}$ melt, we observed a variation of the exponent $m$ from 0.5 to 0.7 with increasing undercooling. This case was found to correlate with a confinement effect of the liquid through the crystalline wall, leading to a diffusion drop at the SL interface.

As mentioned in the Introduction, the comparison of PFC with $\mathrm{MD}$ and $\mathrm{PF}$ can be considered here only for relative quantities, like anisotropy parameters, owing to the different growth kinetics in play, that is, diffusion- versus collisioncontrolled one, respectively. A comparison of the insets of Figs. 3 and 10 shows that the PFC model used here agrees better with the MD results regarding the growth anisotropy than does the PFC model of [25]. The latter yields $\varepsilon_{1} \approx 2$ and $\varepsilon_{2}<1$ for a bcc structure. The MD/PFC agreement in our case, concerning the dynamical properties $\varepsilon_{1}$ and $\varepsilon_{2}$, corroborates the trend shown by Jaatinen et al. in Ref. [21] for thermodynamic and elastic properties of bcc Fe.

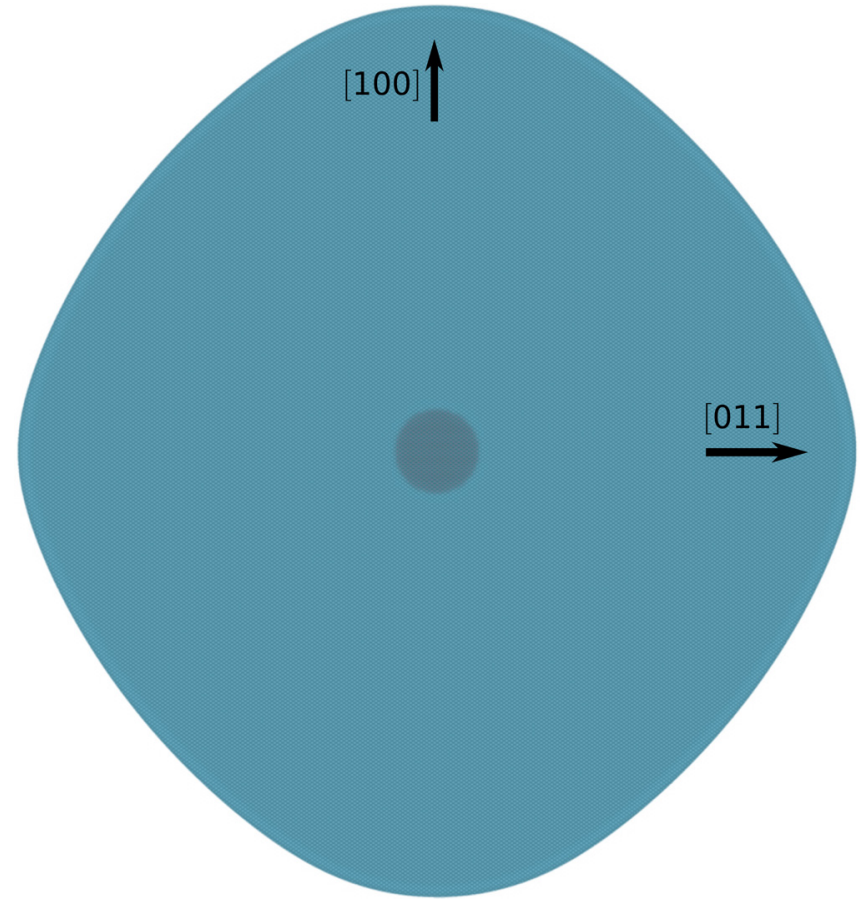

FIG. 12. As in Fig. 12 for a cylinder-axis orientation along [110] (compare with bottom of Fig. 4).

Similar to [25], the solidification velocity is found to increase continuously with $n$ in Fig. 10. We miss here a decrease of the curve slope with increasing $n$. This decrease is known for velocity-versus-undercooling curves, similar to Fig. 3. It follows from the competition between an increasing driving force and a decreasing diffusion as $T$ decreases [26]. The absence of such a behavior in Fig. 10 and in Ref. [25] is likely due to the simplified assumption of a constant (densityindependent) diffusivity $M$ in Eq. (10).

Solidification results by PFC for cylindrical seeds are illustrated by Figs. 11-13. As in Sec. III A, we considered the orientations [100] and [110] of the cylinder axis. These PFC simulations reproduce the fourfold morphology we observed by MD in Fig. 4. The ratio of the growth rates along (100) and (110) fronts is found to be about 1.06 for both cylinder-axis orientations, which is comparable to the growth anisotropy by $\mathrm{MD}$ and PF calculations in Fig. 5 (inset). This is consistent with the fact that MD and the EOF PFC model yield comparable $\gamma$ anisotropies [21].

\section{CONCLUDING REMARKS}

Our study illustrates how the PF approach is able to reproduce different aspects of the growth kinetics deduced from MD simulations when the key physical parameters (structural, thermodynamic, and kinetic) are provided by the latter method to construct the former one. We showed the MD-PF consistency in the case of collision-limited growth for pure Fe. This concerns the growth velocity and growth anisotropy of pseudo-two-dimensional cylindrical seeds. These conclusions extend the ones we did earlier for diffusion-limited growth under chemical nonequilibrium in the binary alloy NiZr [3]. 


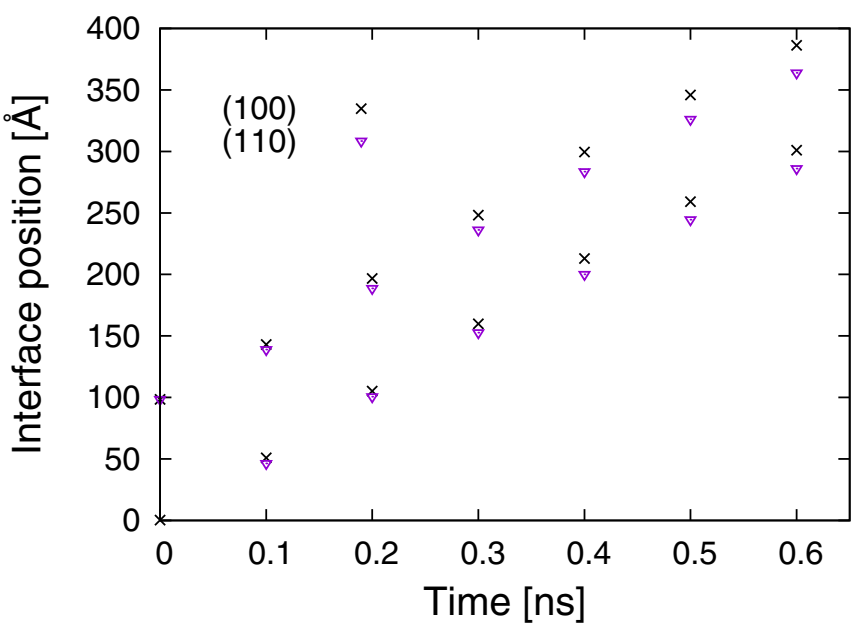

FIG. 13. PFC results of the SL interface position $\Delta R(t)$ along the [100] and [110] directions for a cylinder axis parallel to [100] (lower two curves) and to [110] (upper two curves). All curves should start at the origin. For clarity, the upper two curves have been shifted vertically.

We revisited the kinetic coefficient $\mu$ of pure Fe to account for thermostat issues that have been neglected before and which led to a significant underestimate of $\mu$ by almost a factor of 2 . Furthermore, a special care has to be taken when determining the velocity of curved SL interfaces under isothermal conditions. In order to avoid the formation of temperature-gradient artifacts at the interface during solidification, we divided the simulation box into a lattice of rectangular cells and maintained each one of them at the same target temperature $T$. The volume of one cell must be large enough to describe $T$ properly. After the MD model has been mapped to the PF one, this latter offers the possibility for analyzing the separate contribution of each anisotropy to the dynamics of SL interface morphology. We found, for instance, that the fourfold growth shape of the cylindrical seed is controlled predominantly by the capillary anisotropy of $\gamma(\hat{\mathbf{n}})$. Notice that $\gamma(\hat{\mathbf{n}})$ and its anisotropy in Table II has been calculated from the density-fluctuation profile (i.e., capillarity) of plane SL interfaces with different orientations [8]. These fluctuations at atomic level, present naturally in MD simulations, are absent in the PF model where the $\gamma(\hat{\mathbf{n}})$ anisotropy is incorporated in a phenomenological way. Linking PF to MD modeling permits, on the one hand, to assess this phenomenological construction. On the other hand, it shows that our MD simulations are capable to predict correctly interface-morphology symmetry and dynamics up to $\sim 0.15 \mu \mathrm{m}$.

We showed further that the Fe PFC model of Jaatinen et al. [21] reproduces well the growth anisotropy and morphology deduced by MD simulations. This MD/PFC agreement regarding a dynamical property extends the trend observed in [21] for thermodynamic and elastic properties of bcc Fe. We notice, however, that this agreement is not obvious in view of the fact that PFC describes a diffusion-controlled growth, while MD simulations model a collision-limited solidification process with a constant velocity. Moreover, Tegze et al. [27] pointed out that MD models represent materials of low melting entropy leading to an extended SL interface (4-5 atomic layers), whereas PFC tends to realize a sharp interface. Nevertheless, our result confirms the possibility of the EOF model to be used in PFC studies where the quantitative accuracy of the results is desired [21]. Such a possibility could be useful when we know that the PFC approach generally lacks accuracy in reproducing physical properties of the system to which the energy functional has been fitted [28]. We notice that a better quantitative comparison of MD and PFC growth modeling would be, in principle, possible when performing the MD simulations in a NVT (instead of NPT) thermodynamic ensemble which is the natural ensemble of PFC methods. The growth kinetics would be then diffusion controlled, in MD as well as in PFC simulations.

Our study illustrates how atomistic MD modeling, with the increasing computational capabilities, is progressing beyond its status as "parameters provider" to become a framework for validating the accuracy of solidification theories and phenomenological modeling approaches.

\section{ACKNOWLEDGMENTS}

M.G. thanks O. Tschukin for helpful discussions and C. Ratz for his "critical eye" on the growth morphology. This work was supported by the German Research Foundation (DFG) under Grants No. GU 1258/2-1 and No. GU 1258/2-2.
[1] J. J. de Pablo and W. A. Curtin, MRS Bull. 32, 905 (2007).

[2] W. A. Curtin and R. E. Miller, Modell. Simul. Mater. Sci. Eng. 11, R33 (2003).

[3] M. Guerdane, F. Wendler, D. Danilov, H. Teichler, and B. Nestler, Phys. Rev. B 81, 224108 (2010).

[4] M. Guerdane, H. Teichler, and B. Nestler, Phys. Rev. Lett. 110, 086105 (2013).

[5] M. Asta, C. Beckermann, A. Karma, W. Kurz, R. Napolitano, M. Plapp, G. Purdy, M. Rappaz, and R. Trivedi, Acta Mater. 57, 941 (2009)

[6] J. Bragard, A. Karma, Y. Lee, and M. Plapp, Interface Sci. 10, 121 (2002).

[7] M. Berghoff, M. Selzer, and B. Nestler, Sci. World J. 2013, 564272 (2013).
[8] D. Y. Sun, M. Asta, and J. J. Hoyt, Phys. Rev. B 69, 174103 (2004).

[9] K. R. Elder, N. Provatas, J. Berry, P. Stefanovic, and M. Grant, Phys. Rev. B 75, 064107 (2007).

[10] M. I. Mendelev, S. Han, J. Srolovitz, G. J. Ackland, D. Y. Sun, and M. Asta, Philos. Mag. 83, 3977 (2003).

[11] D. Y. Sun, M. Asta, and J. J. Hoyt, Phys. Rev. B 69, 024108 (2004).

[12] J. Monk, Y. Yang, M. I. Mendelev, M. Asta, J. J. Hoyt, and D. Y. Sun, Modell. Simul. Mater. Sci. Eng. 18, 015004 (2010).

[13] M. J. Uline and D. S. Corti, Entropy 15, 3941 (2013).

[14] H. Garcke, B. Nestler, and B. Stinner, SIAM J. Appl. Math. 64, 775 (2004).

[15] W. R. Fehlner and S. H. Vosko, Can. J. Phys. 54, 2159 (1976). 
[16] W. J. Boettinger, J. A. Warren, C. Beckermann, and A. Karma, Annu. Rev. Mater. Sci. 32, 163 (2002).

[17] See Supplemental Material at http://link.aps.org/supplemental/ 10.1103/PhysRevB.97.144105 for the growth velocity around $T_{m}$ for three interface orientations.

[18] Y. F. Gao, Y. Yang, D. Y. Sun, M. Asta, and J. J. Hoyt, J. Cryst. Growth 312, 3238 (2010).

[19] Y. Shibuta, K. Oguchi, and M. Ohno, Scripta Mater. 86, 20 (2014).

[20] H. E. A. Huitema, M. J. Vlot, and J. P. van der Erden, J. Chem. Phys. 111, 4714 (1999).

[21] A. Jaatinen, C. V. Achim, K. R. Elder, and T. Ala-Nissila, Phys. Rev. E 80, 031602 (2009).
[22] K.-A. Wu and A. Karma, Phys. Rev. B 76, 184107 (2007).

[23] G. Tegze, G. Bansel, G. I. Tóth, T. Pusztai, Z. Fan, and L. Gránásy, J. Comput. Phys. 228, 1612 (2009).

[24] S. Tang, R. Backofen, J. Wang, Y. Zhou, A. Voigt, and Y.-M. Yu, J. Cryst. Growth 334, 146 (2011).

[25] G. Tegze, L. Gránásy, G. I. Tóth, F. Podmaniczky, A. Jaatinen, T. Ala-Nissila, and T. Pusztai, Phys. Rev. Lett. 103, 035702 (2009).

[26] K. A. Jackson, Interface Sci. 10, 159 (2002).

[27] G. I. Tóth, G. Tegze, T. Pusztai, G. Tóth, and L. Gránásy, J. Phys.: Condens. Matter 22, 364101 (2010).

[28] M. Oettel, S. Dorosz, M. Berghoff, B. Nestler, and T. Schilling, Phys. Rev. E 86, 021404 (2012). 